\title{
A light, scanning electron and transmission electron microscopic study of a Fetal mouse Skin Dendritic Cell line (FSDC)
}

\author{
A. M. Dinis****, M. T. Cruz***, M. C. Lopes*** and M. T. Batista* \\ * Laboratory of Pharmacognosy (CEF), Faculty of Pharmacy, University of Coimbra, 3000-548 \\ Coimbra, Portugal. \\ ** Laboratory of Electron Microscopy, Department of Botany, Faculty of Sciences and Technology, \\ University of Coimbra, 3001-455 Coimbra, Portugal. \\ *** Centre for Neurosciences and Cell Biology (CNC) and Faculty of Pharmacy, University of \\ Coimbra, 3004-517 Coimbra, Portugal. \\ amdinis@bot.uc.pt
}

Dendritic cells (DC) are major players in initiating immune responses by activating T-lymphocytes. They act as sentinels in peripheral tissues, continuously scavenging for antigens in their immediate surroundings. Their involvement in T-cell responses consists of a linear progression of events starting with capture of antigens in peripheral tissues, such as the skin, followed by migration to draining lymphoid organs and presentation of antigen-derived peptides to induce T-cell priming [1]. In the last years, a number of DC lines have been generated and the electron microscopy has been used to define their characteristics, thus contributing to their formal validation. A cell line with features of early DC precursors was previously established from fetal mouse skin (FSDC) [2], but its ultrastructural organization was not fully investigated. Here we report such investigation using light (LM), scanning electron (SEM) and transmission electron microscopy (TEM). The goal is to use FSDC as a model system to evaluate the safety and effectiveness of selected plant extracts, fractions and isolated polyphenols against inflammatory processes. Our preliminary results with these cells showed anti-inflammatory activity for some of the extracts we are currently investigating [3].

Pelleted FSDC cells were conventionally fixed in glutaraldehyde and osmium tetroxide. For SEM examination the cells were further dehydrated in ethanol, critical point dried and sputter coated with gold-palladium. For TEM examination they were post-fixed in $1 \%$ aqueous uranyl acetate for $1 \mathrm{~h}$, dehydrated in ethanol and embedded in Spurr's resin. In addition, a sample of cells was cytochemically tested for acid phosphatase activity. For LM examination, thin sections were stained with toluidine blue, or else with Sudan black B to detect lipid bodies.

FSDC cells are spherical, or more or less elongated in shape, with numerous dendrite-like projections on the cell surface (Figs. 1 and 2). The nucleus is large, lobed, peripherally located, with a few dense masses of heterochromatin and two large nucleoli consisting of a variable number of small fibrillar centres and an especially well-developed granular region (Fig. 3). In the cortical cytoplasm a number of coated pits and vesicles are frequently found (Figs. 3 and 6). Throughout the cytoplasm the mitochondria are abundant with a highly electron-dense matrix containing many electron-transparent packed cristae (Figs. 3 and 6). A variable number of lipid bodies and Golgi stacks formed by swollen cisternae are also found as well as single and stacked rough endoplasmic reticulum (RER) cisternae having a relatively reduced, electron-lucent lumen (Fig. 6). On occasion, some single ER cisternae are seen consisting of two distinct regions in perfect continuity, i.e. one provided with membrane-bound ribosomes and with the lumenal space relatively reduced, and the other one deprived of ribosomes with the electron-transparent lumenal space either very reduced or 
reasonably enlarged (Figs. 3 and 6). In addition, cup-shaped vacuolar structures, presumably autophagosomes, are frequently seen sequestering portions of the cytosol and organelles (Fig. 4). The most characteristic feature of these cells is the extremely high number of polymorphic vacuolar structures, which contain diverse kind of material in various stages of degradation (Figs. 4 and 6). Following the cytochemical test for acid phosphatase activity, many of these vacuolar structures showed the electron-opaque reaction product deposited in the matrix (Fig. 5) indicating that they are lysosomes. No other organelles have shown a positive reaction. The staining reaction did not occur at all when $\beta$-glycerophosphate was absent or the inhibitor sodium fluoride was added to the medium of incubation. Clearly, FSDC cells display morphologic and structural features of DC making them a useful in vitro model system for in-depth studies related to innate immune responses.

\section{References}

[1] G.J. Randolph, V. Angeli, M.A. Swartz. Nat Rev Immunol, 5 (2005) 617.

[2] G. Girolomoni, M.B. Lutz, S. Pastore et al. Eur J Immunol, 25 (1995) 2163.

[3] A.M. Figueirinha, M.T. Cruz, M.C. Lopes, M.T. Batista. In: F. Daayf, A. EL Hadrami, L. Adam, G. M. Ballance (Eds.), Polyphenols Communications 2006, Winnipeg, Canada, 2006, p 499.
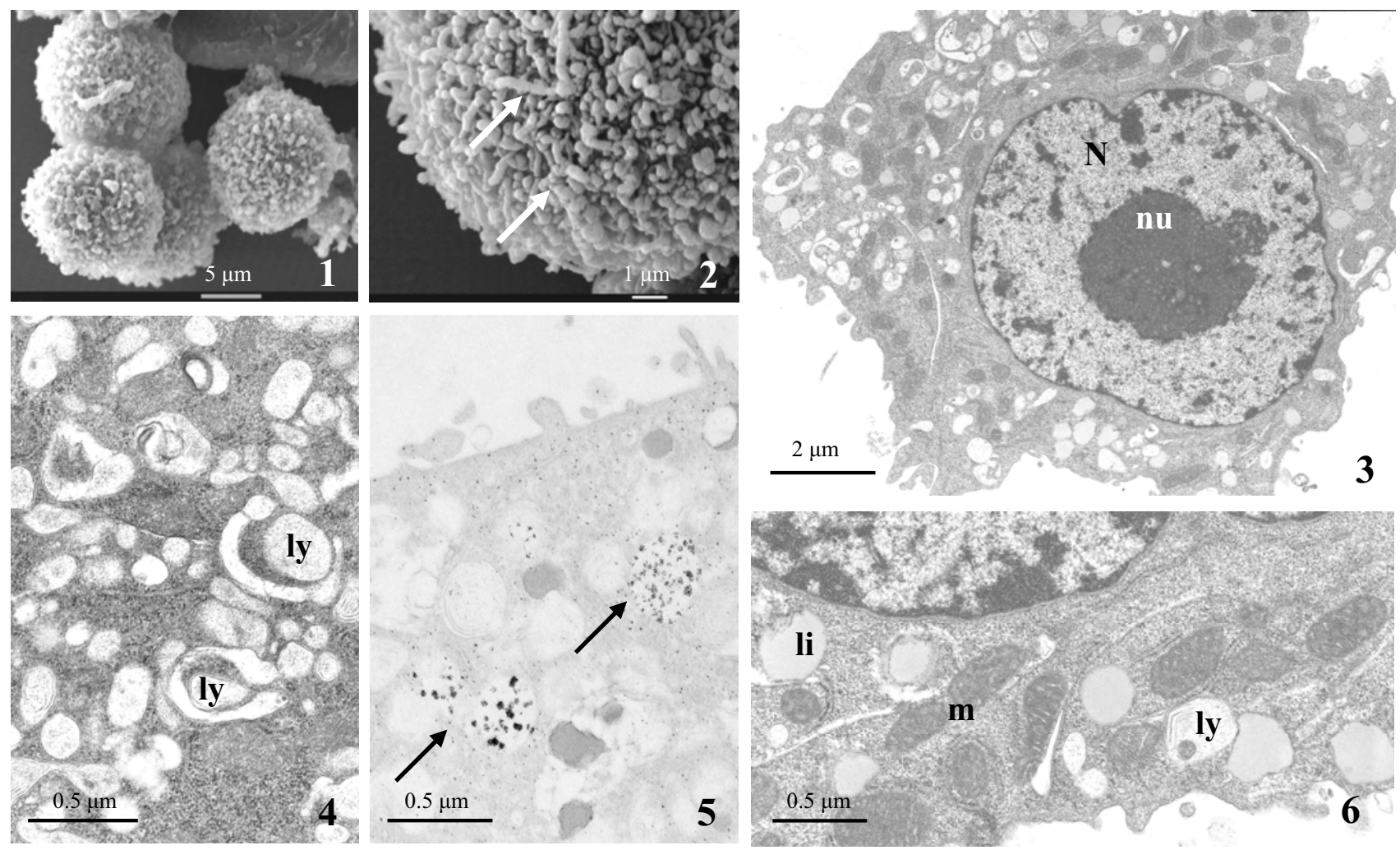

Figs. 1 and 2. SEM micrographs of FSDC cells showing numerous dendrite-like projections (arrows) on the cell surface.

Fig. 3. TEM micrograph of a FSDC cell showing the nucleus $(N)$ with a large nucleolus $(n u)$.

Fig. 4. TEM micrograph of a portion of cytoplasm containing numerous lysosomes $(l y)$.

Fig. 5. TEM micrograph showing the cytochemical localization of acid phosphatase activity; note the electron-dense reaction product deposited within the vacuoles (arrows).

Fig. 6. TEM micrograph showing details of mitochondria $(m)$, lysosomes $(l y)$ and lipid bodies $(l i)$. 